\title{
PROPUESTA DE UN PROYECTO PARA DISMINUIR LOS ALTOS ÍNDICES DE VIOLENCIA Y DELINCUENCIA EN LA PROVINCIA DE LA ROMANA, REPÚBLICA DOMINICANA
}

\section{Proposal for a project to reduce the high indices of violence and crime in the province of La Romana, Dominican Republic}

\author{
Magnolia Ortiz Rodríguez \\ Candidata a doctora en Proyectos de la Universidad Internacional Iberoamericana (UNINI). México \\ magnolia_ortiz06@hotmail.com
}

Recibido: 8/8/2018 • Aprobado: 10/9/2018

Cómo citar: Ortiz Rodríguez, M. (2018). Propuesta de un proyecto para disminuir los altos índices de violencia y delincuencia en la provincia de La Romana, República Dominicana. Ciencia y Sociedad, 43(3), 41-55. doi:http://dx.doi.org/10.22206/cys.2018. v43i3.pp41-55

\section{Resumen}

El objetivo de esta investigación es hacer un diagnóstico de la violencia y la delincuencia en la provincia de La Romana en el año 2017. Metodología: Es un estudio descriptivo en el cual se utilizan datos secundarios de la Fiscalía de La Romana; datos de informes de trabajos de campo en destacamentos preventivos y se realizan estimaciones sobre el costo de la delincuencia a través del método contable. Se utiliza la observación y análisis. En éste se analiza el nivel de violencia y delincuencia. Se verifica el porcentaje de presos preventivos y condenados, se calcula la tasa de hacinamiento carcelario y se estima el costo del sistema judicial y penitenciario. Los hallazgos muestran que La Romana tiene un elevado índice de violencia y delincuencia; la tasa de homicidios de $24 \%$ por cada 100,000; el robo, una tasa de $110 \%$ por 100,000 habitantes. Se revela que solo el $15 \%$ de los presos estaban condenados y el $85 \%$ eran preventivos. Se encontró hacinamiento crítico en el destacamento del Palacio de Justicia; con una tasa entre 320\% y 490\%. Se calculó que el consto en el sistema penal y penitenciario fue de RD\$ 763,332,360, equivalente a USD15.902.757.

Palabras claves: Violencia; delincuencia; costo; hacinamiento, República Dominicana.

\begin{abstract}
The objective of this research is to make a diagnosis of violence and crime in the province of La Romana in 2017. Methodology: It is a descriptive study in which secondary data from the La Romana Prosecutor's Office are used; data from reports of field work in preventive detachments and estimates are made about the cost of crime through the accounting method. Observation and analysis are used. In this one the level of violence and delinquency is analyzed. The percentage of pre-trial and convicted prisoners is verified, the rate of prison overcrowding is calculated and the cost of the judicial and penitentiary system is estimated. The findings show that La Romana has a high rate of violence and delinquency; the homicide rate of $24 \%$ per 100,000 ; theft, a rate of $110 \%$ per 100,000 population. It is revealed that only $15 \%$ of the prisoners were convicted and $85 \%$ were preventive. Critical overcrowding was found in the detachment of the Palace of Justice; with a rate between 320\% and $490 \%$. It was calculated that the cost in the penal and penitentiary system was $\mathrm{RD} \$ 763,332,360$, equivalent to USD15,902,757.
\end{abstract}

Keywords: Violence; crime; cost; overcrowding, Dominican Republic. 


\section{Introducción}

La Organización Mundial de la Salud define la violencia como el uso deliberado de la fuerza física o el poder, ya sea en grado de amenaza o efectivo, contra uno mismo, otra persona o un grupo o comunidad, que cause o tenga muchas probabilidades de causar lesiones, muerte, daños psicológicos, trastornos del desarrollo o privaciones. El delito es se refiere al conjunto de conductas ilegales que son contrarias a las leyes o normas de un Estado y que trae como consecuencia el castigo o sanción (Programa de las Naciones Unidas para el Desarrollo, 2014).

La violencia y la delincuencia se diferencian en que puede cometerse delito sin violencia y actos de violencia no considerarse como delito; no obstante, ambas constituyen amenazas a la vida y a la integridad física y patrimonial de las personas. En tal sentido, la alta tasa de violencia y delincuencia registrado en un país o región afecta directa o indirectamente la seguridad de los ciudadanos; ya sea por el hecho de ser víctima o debido a factores psicológicos y conductuales producidos por el miedo a ser víctima. En ese orden, la inseguridad ciudadana puede tener dos implicaciones; objetiva y subjetiva.

La inseguridad objetiva se mide mediante estadísticas de hechos delictivos que se cometen en una comunidad, cuantificando la cantidad de denuncias, el tipo de delitos, datos demográficos de las víctimas y los victimarios. También se realizan encuestas sobre la victimización, que permite medir la cantidad de víctimas o "cifras ocultas" de personas que no denunciaron. (Instituto Interamericano de Derechos Humanos, IIDH, 2011) Por otro lado, la inseguridad subjetiva se establece con base a la percepción de la delincuencia y el miedo a la violencia y al delito de los habitantes.

Se ha determinado que para disminuir la violencia y la delincuencia es necesario desarrollar políticas de prevención y que éstas se apliquen en los tres niveles de intervención; primario, secundario y terciario. Sin embargo, los estudios revelan que el apresamiento es la medida más utilizada en los países de América Latina y El Caribe por lo cual las cárceles están sobrepobladas. La sobrepoblación penitenciaria trae como consecuencia una serie de privaciones para satisfacer necesidades básicas de sobrevivencia, además, el contagio de enfermedades, complicaciones de salud, violencia, enfermedades mentales y poco o nada actividades de ocio y abuso sexual entre reclusos.

La alta tasa de violencia y delincuencia y la sobrepoblación penitenciaria, representan un alto costo en la economía. Según estudios del Banco Interamericano de Desarrollo, los gastos del gobierno destinado a la delincuencia se han incrementado durante varios años; el gasto público en la administración penitenciaria de América Latina y El Caribe en 2010 fue de US\$ 4.318 millones y en 2014 se duplicó a US\$ 7.832 millones y se estima que la pérdida por inactividad de los encarcelados fue un promedio de US\$7.000 millones desde 2010 a 2014.

Este artículo pretende analizar la tasa de violencia y delincuencia en la provincia de La Romana en 2017, indagar sobre la sobrepoblación en cuartel preventivo y calcula el costo de los privados de libertad en el Centro de Corrección y Rehabilitación y del sistema de justicia penal. Los resultados servirán como base para proponer un proyecto de prevención de la violencia y la delincuencia.

\section{La violencia y la delincuencia en América Latina y El Caribe}

Como se expuso anteriormente, no todo delito es violento ni todo acto de violencia es tipificado como delito en las leyes. (Programa de las Naciones Unidas para el Desarrollo, 2014). La Organización Mundial de la Salud ${ }^{1}$, define la violencia como:

\footnotetext{
1. Organización Mundial de la Salud, 2014, Informe sobre la situación mundial de la prevención de la violencia. Tomado de apps.who.int/iris/bitstream/10665/145089/1/WHO_NMH_ NVI_14.2_spa.pdf
} 
El uso deliberado de la fuerza física o el poder, ya sea en grado de amenaza o efectivo, contra uno mismo, otra persona o un grupo o comunidad, que cause o tenga muchas probabilidades de causar lesiones, muerte, daños psicológicos, trastornos del desarrollo o privaciones. (Organización Mundial de la Salud, 2014).

A pesar de que existe diferencia entre el delito y la violencia, se ha detallado que ambas representan una amenaza a la seguridad ciudadana. En ese sentido, la inseguridad ciudadana puede tener dos implicaciones; objetiva y subjetiva. La inseguridad objetiva se mide mediante estadísticas de hechos delictivos que se cometen en una comunidad, cuantificando la cantidad de denuncias, el tipo de delitos, datos demográficos de las víctimas y los victimarios. También se realizan encuestas sobre la victimización, que permite medir la cantidad de víctimas o "cifras ocultas" de personas que no denunciaron. (Instituto Interamericano de Derechos Humanos, IIDH, 2011). La inseguridad subjetiva se establece con base a la percepción de la delincuencia y el miedo a la violencia y al delito de los habitantes. Con base esto, la encuesta de Latinobarómetro revela que el $88 \%$ de los ciudadanos latinoamericano dijo sentir temor de ser víctima de un delito. (Latinobarómetro, 2016).

Sobre la victimización, estudios revelan que América es la más violenta del mundo; según la Organización Mundial de la Salud, "se estima que en 2012 hubo 475,000 muertes por homicidio en todo el mundo y la Región de las Américas, obtuvo la mayor cantidad por cada 100.000 habitantes". (Organización Mundial de la Salud, 2014). Otros estudios indican que dentro de las 50 ciudades más violentas del mundo, en el año 2017; 46 de éstas pertenecen a la región de América Latina y El Caribe, situando a la ciudad de Los Cabos, México, con el número uno, con una tasa de $111.33 \%$ por cada 100,000 habitantes, y la ciudad de Cúcuta, Colombia, en el número 50, con una tasa de
$34.78 \%$ por cada 100,000 habitantes. (Seguridad, Justicia y Paz, 2018)2. Cabe resaltar que "el homicidio constituye uno de los indicadores más completos, comparables y precisos para medir la violencia". (Oficina de las Naciones Unidas contra la Droga y el Delito, 2014).

Se estima que una tasa superior a 10 homicidios por cada 100.000 habitantes tiene carácter epidémico. (Centro de las Naciones Unidas Regional para la Paz, el Desarme y el Desarrollo en América Latina y el Caribe, 2017); una tasa normal se encuentra ente 0 a 5 homicidios por cada 100,000 habitantes. (Kliksberg, 2007)

El propósito de medir el nivel de violencia es revelar la realidad sobre la criminalidad y generar acciones de prevención y disminución de la misma. La Oficina de las Naciones Unidas contra la Droga y el Delito $^{3}$, UNODC, 2013 identifica tres tipologías de homicidios; según la motivación, según el contexto y según el vínculo entre víctima y victimario. En primer lugar, están los homicidios vinculado a otras actividades delictivas, dentro de ellos están la delincuencia organizada y las pandillas. En estos se encuentran los homicidios por robo o atraco, la trata de personas y los homicidios ligados a las drogas.

En segundo lugar, se encuentran los homicidios asociados a conflictos interpersonales: se derivan de la intención de resolver un conflicto o castigar a la víctima mediante la violencia cuando las relaciones se tensan. "Desde el 2000, han muerto en todo el mundo cerca de 6 millones de personas en actos de violencia interpersonal". (Organización Mundial de la Salud, 2016). Dentro de este tipo se encuentra la

2. Seguridad, Justicia y Paz, 2018. Metodología del ranking (2017) de las ciudades más violentas del mundo. México. Recuperado de https://www.seguridadjusticiaypaz.org.mx/biblioteca/ prensa/category/6-prensa

3. Oficina de las Naciones Unidas contra la Droga y el Delito, Estudio Mundial sobre el Homicidio 2013. Recuperado de https://www.unodc.org/documents/gsh/pdfs/GLOBAL_HOMICIDE_Report_ExSum_spanish.pdf 
violencia intrafamiliar, cuyas víctimas son, con más frecuencia, las mujeres, niños, niñas y ancianos.

En tercer lugar están los homicidios relacionado con motivos sociopolítico: ocurre cuando se intenta ejercer influencia en las relaciones de poder y para impulsar una agenda en particular. Dentro de esta categoría se encuentra el terrorismo, y los asesinatos relacionados con la guerra y el conflicto. (Oficina de las Naciones Unidas contra la Droga y el Delito, UNODC, 2013).

\section{Sobrepoblación carcelaria}

A pesar que la violencia y la delincuencia son problemas con múltiples causas y que requieren estrategias multidisciplinarias para enfrentarlas; no obstante, la privación de libertad es la medida más usada en los países de América Latina y El Caribe. En ese sentido, se ha determinado que el encarcelamiento no resulta ser efectivo ni eficaz, y tampoco reduce el delito ni la violencia.

Se ha encontrado que América Latina y El Caribe, poseen indicadores de violación de los derechos humanos de los privados de libertad, causada fundamentalmente por la sobrepoblación penitenciaria. (Comisión Interamericana de Derechos Humanos, 2011). La sobrepoblación penitenciaria se refiere

Situación en que la densidad penitenciaria es mayor que 100, porque hay más personas presas que la capacidad establecida para una prisión o para la totalidad del sistema; cuando la densidad es igual o mayor que 120 , se considera sobrepoblación crítica o hacinamiento crítico." (Carranza, 2012).

Las consecuencias del hacinamiento tienen diferentes repercusiones; en algunos centros de

4. Carranza, 2012. Situación penitenciaria en América Latina y el Caribe ¿Qué hacer? Chile. Anuario de Derechos Humanos. Recuperado de https://anuariocdh.uchile.cl/index.php/ADH/ article/download/20551/21723/0 reclusión el nivel de hacinamiento puede ser tan agudo que fuerza a los reclusos a dormir por turnos, o encima uno de otro. (Naciones Unidas, 2014). ${ }^{5}$

La sobrepoblación penitenciaria en República Dominicana presenta niveles críticos en las cárceles públicas del país. La tabla a continuación presenta la tasa de hacinamiento en 2014 según la Oficina Nacional de la Defensa Pública.

\section{Tabla 1 Sobrepoblación penitenciaria en el modelo tradicional de República Dominicana en 2014}

\begin{tabular}{cc}
\hline Sobrepoblación & \% de hacinamiento \\
\hline Cárcel La Victoria & 320 \\
Cárcel del Seybo & 374 \\
La Vega & 332 \\
Pedernales & 312 \\
San Juan & 440 \\
Neyba & 216 \\
Barahona & 228 \\
15 de Azua & 166 \\
19 de Azua & 402 \\
Monte Cristi & 570 \\
Operaciones Especiales & 400 \\
Higuey-Mujeres & 112 \\
Santiago Rodríguez & 385 \\
Baní & 535 \\
Nagua & 357 \\
Cotuí & 405 \\
Salcedo & 216 \\
Fortaleza Duarte & 137 \\
\hline
\end{tabular}

Fuente: Elaboración propia, basado en (Oficina Nacional de Defensa Pública, 2014)

Como muestra la tabla, el nivel de sobrepoblación penitenciaria en el año 2014, fue extremadamente crítica, siendo la cárcel Higuey-mujeres la que presenta el nivel más bajo, con $112 \%$, y la cárcel

5. Naciones Unidas, 2014. Manual Sobre Estrategias Para Reducir el Hacinamiento en las Prisiones. New York. UNODC. 
de Montecristi el nivel más alto con 570\%. Cabe resaltar que este estudio no refleja la sobrepoblación en los destacamentos preventivos.

Se ha determinado que la causa fundamental de la sobrepoblación penitenciaria se debe al uso excesivo de la prisión preventiva, en ese sentido, la prisión preventiva, que debería ser utilizada como último recurso, sin embargo, se volvió una pena anticipada y sobre utilizada 6 . (Barros Leal, 2014).

Por otro lado, se ha señalado que la sobrepoblación carcelaria se debe al rezago judicial de los expedientes de gran parte de la población en reclusión; casi el $50 \%$ son procesados (Comisión Nacional de los Derechos Humanos , 2015) ${ }^{7}$. Lo anterior descrito, es confirmado por María Noel Rodríguez ${ }^{8}$, la cual añade, que el hacinamiento se debe a la ineficacia del sistema de justicia (Noel Rodríguez, 2015).

Cabe destacar que el hacinamiento carcelario es evidente tanto en adultos y en menores de edad. En tal sentido, el Informe de monitoreo a los sistemas de justicia penal juvenil en América latina ${ }^{9}$ manifiesta que "el hacinamiento se constituye en una forma de trato cruel, inhumano y degradante contra la integridad de adolescentes y jóvenes, es una seria problemática en los centros de privación de libertad" (Observatorio Regional de Justicia Penal Juvenil, 2015).

6. Barros Leal, 2014. La Pena de la Prisión en América Latina: Los Privados de Libertad y sus Derechos. Revista del Instituto Brasileño de Derechos Humanos. http://milas.x10host.com/ojs/ index.php/ibdh/article/view/265

7. Comisión Nacional de los Derechos Humanos, 2015. La Sobrepoblación en los Centros Penitenciarios de la República Mexicana. Recuperado de http://www.cndh.org.mx/sites/all/doc/ Informes/Especiales/Pronunciamiento_20151014.pdf

8. Noel Rodríguez, 2015. Hacinamiento Penitenciario En América Latina: Causas Y Estrategias Para Su Reducción. México, D.F. Comisión Nacional de los Derechos Humanos

9. Observatorio Regional de Justicia Penal Juvenil, 2015. Informe de monitoreo a los sistemas de justicia penal juvenil en América latina. Bolivia. Recuperado de www2.congreso.gob.pe/.../ CARTILLA-ESPAÑOL-DNI-Congreso-ONU-Qatar.pdf

\section{Costo de la delincuencia en América Latina y El Caribe}

La consecuencia de la violencia y la delincuencia repercuten en la economía de los Estados. Con base a esto "los costos directos están directamente vinculados con el crimen y la violencia, cuyos resultados son daños a la propiedad y lesiones a las víctimas" (Banco Interamericano de Desarrollo, 2015).

Para cuantificar el costo directo se debería calcular la pérdida material y las lesiones de la víctima, la consecuencia de la discapacidad, días de trabajo perdido y la vida productiva de quienes han muerto. Otros costos que se estiman son la atención médica, costos institucionales como seguridad pública y justicia, costos de seguridad privada, impactos negativos sobre las inversiones externas, pérdida de oportunidades de trabajo y pérdidas materiales. (Kliksberg, 2007)

Los costos también se adjudican al delincuente, ya que involucra los costos de las actuaciones policiales, del sistema de justicia penal, la administración carcelaria y productividad no percibida de la persona privada de libertad (Banco Interamericano de Desarrollo, 2017).

Se ha valorado que el gasto público en la administración penitenciaria de América Latina y El Caribe en 2010 fue de US\$ 4.318 millones y en 2014 se duplicó a US $\$ 7.832$ millones y se estima que la pérdida por inactividad de los encarcelados fue un promedio de US\$ 7.000 millones desde 2010 a 2014 (Banco Interamericano de Desarrollo, 2017).

Otro aspecto importante radica en que en algunos países el costo por interno diario supera el sueldo mínimo de un empleado. En ese orden, en Estados Unidos, el sueldo mínimo era 28.3 dólares diarios, mientras que el costo diario por recluso era de 65 dólares diarios en 2003; en Inglaterra, el sueldo mínimo era 67,4 dólares diarios y el costo de 
encarcelamiento por recluso era de 166 dólares; asimismo, en Australia el diario era de 63,4 y el sueldo mínimo era de 84 dólares diarios en 2003 $\left(\right.$ Dammert \& Díaz, 2005) ${ }^{10}$.

El costo de una persona privada de libertad supera el realizar una carrera universitaria. En ese orden, se determinó que en 2002 el costo de una carrera universitaria por persona en 15 estados de Estados unidos era de 9.454 dólares en un año, mientras que el costo de un año de reclusión por persona era de 24.809 dólare. (Dammert \& Díaz, 2005).

Uno de los métodos utilizados para estimar el costo de la delincuencia es el enfoque contable. Esta aproximación busca agregar los gastos monetarios directos asociados a la inseguridad; entre ellos, se incluye una valoración de las pérdidas y de los daños asociados al crimen, así como los gastos de contención, atención y prevención (Programa de las Naciones Unidas para el Desarrollo, 2014).

Un problema de este método, sin embargo, además de demandar información administrativa muy detallada sobre gastos públicos y encuestas de gasto de familias, es que puede llevar a errores de doble imputación (por ej. los gastos médicos en parte ya podrían estar incluidos en las compensaciones determinadas por la justicia). (Corporación Andina de Fomento, 2014)

\section{Metodología}

La provincia La Romana forma parte de la región Yuma y cuenta con una superficie de $652.12 \mathrm{~km}^{2}$. Limita al norte con la provincia El Seibo, al este con La Altagracia, al sur con el mar Caribe y al oeste con las provincias El Seibo y San Pedro de Macorís.

10. Dammert \& Díaz, 2005. El Costo de Encarcelar. Programa de Seguridad y Ciudadanía FLACSO-Chile. Observatorio No9, noviembre. Recuperado de http://www.flacsochile.org/wp-content/uploads/2015/05/El-costo-de-Encarcelar.pdf
Está conformada por tres municipios, dos distritos municipales. Según el IX Censo Nacional de Población y Vivienda 2010, La Romana contaba para ese año con una población de 245,433 habitantes (Oficina Nacional de Estadísitica, 2016).

De acuerdo al informe del Ministerio de Educación, en la provincia de La Romana, hubo una inversión promedio de $\mathrm{RD} \$ 49,415$ por estudiante en las escuelas del sector público; siendo ésta una de las provincias que menos se invirtió en la educación por alumno en 2017 (Ministerio de Educación, MINERD, 2018).

Se ha indicado que el problema mayor de esta provincia es la inseguridad, la cual representa un obstáculo para el desarrollo (Ministerio de Economía, Planificación y Desarrollo, 2016).

A pesar que se ha identificado la inseguridad como el problema primordial, sin embargo, no se ha realizado estudios que determinen cuál es el nivel de violencia y delincuencia.

\section{Objetivo general}

Diagnosticar la situación de violencia y delincuencia para proponer un proyecto de prevención en la provincia de La Romana.

\section{Objetivos específicos}

1. Determinar la tasa de violencia y delincuencia en la provincia de La Romana en 2017.

2. Verificar si se respetan las normas de Derechos Humanos a los privados de libertad.

3. Estimar el costo en el sistema de justicia penal y penitenciaria.

\section{Diseño metodológico}

La investigación es descriptiva: Obtiene los datos, analiza y describe la información (Arias, 2012). Por otro lado, se trata de una investigación cuantitativa 
porque recopila, procesa y analiza datos que se pueden contar, cuantificar y medir. (Muñoz Razo, 2011). ${ }^{11}$

La tabla a continuación presenta la variable nivel de violencia y delincuencia y su dimensión e indicadores.

\section{Tabla 2 Variable nivel de violencia y} delincuencia

\begin{tabular}{lll}
\hline Variable & Dimensión & Indicadores \\
\hline Nivel de & Tasa de & . Tasa de violencia y \\
violencia y & delitos & delincuencia \\
delincuencia & & $\cdot$ Homicidios \\
& & $\cdot$ Delitos de droga \\
& & $\cdot$ Robo \\
& & Violencia \\
& & intrafamiliar \\
& & Delitos sexuales \\
\hline
\end{tabular}

Fuente: elaboración propia de la investigación.

Como muestra la tabla anterior, se analiza la variable nivel de violencia y delincuencia. Para medir esta variable se utilizaron los datos secundarios del informe de la Fiscalía de La Romana correspondiente a 2017, en el cual se determinó la cantidad de casos ingresados al sistema, se detalló la cantidad de los diferentes tipos penales y el nivel de delitos por cada 100,000 habitantes. Se estima la tasa de delitos cometidos contra la persona o propiedad.

La tasa de violencia se calcula con la siguiente fórmula:

$\mathrm{THa}=(\mathrm{NTHa} / \mathrm{TP})^{*} 100$ MIL Hbtes ${ }^{12}$

Donde, THa: tasa de homicidios

NTHa: número total de homicidios

TP: población total.

11. Muñoz Razo, 2011. Cómo elaborar y asesorar una investigación de tesis. México. Pearson Educación.

12. Tomado de Organización de los Estados Americanos, 2018. Indicadores sobre Crimen y Violencia. Recuperado de http:// www.oas.org/dsp/espanol/cpo_observatorio_glosario_crimenyviolencia.asp
Total población = 245,433 habitantes.

En la tasa de delincuencia, se utilizó la fórmula a continuación:

$\mathrm{TH}=(\mathrm{NTD} / \mathrm{TP})^{*} 100$ MIL Hbtes.

Dónde: $\mathrm{NTD}=$ número total de Delito.

$\mathrm{TP}=$ número total de la población.

\section{Variable sobrepoblación carcelaria}

La tabla siguiente muestra la dimensión y los indicadores para medir la sobrepoblación carcelaria.

Tabla 3. Variable sobrepoblación carcelaria

\begin{tabular}{lll}
\hline Variable & Dimensión & Indicador \\
\hline Sobrepoblación & Condiciones de & Porcentaje de \\
carcelaria & los privados de & presos preventivos y \\
& libertad & $\begin{array}{l}\text { condenados. } \\
\text { Nivel de } \\
\text { hacinamiento. }\end{array}$ \\
\hline
\end{tabular}

Fuente: elaboración propia de la investigación

La tabla anterior muestra los indicadores que se utilizaron para medir la sobrepoblación y condición de los privados de libertad. Estos datos se tomaron de los informes de levantamientos para verificar las condiciones de los preventivos en los destacamentos y el respeto de los Derechos humanos realizado por el Equipo Técnico de la Oficina de la Defensa Pública del Distrito Judicial de La Romana. Se verificó la condición de hacinamiento en el cuartel preventivo del Palacio de Justicia desde el 2013 hasta 2017. La sobrepoblación se calculó con la siguiente fórmula:

TH = NTPP*100 / OPC; donde, NTPP: población carcelaria total OPC: capacidad carcelaria.

Para obtener el porcentaje de presos preventivos y condenados, se calculó la cantidad de privados de libertad en el Centro de Corrección y Rehabilitación, CCR-15 y en el cuartel preventivo del Palacio de Justicia con la siguiente fórmula: 
$\sum$ TPP -TPC; donde:

TPP: Total de presos preventivos

TPC: Total de presos condenados.

Variable costo de la violencia y la delincuencia

La tabla siguiente muestra la variable costo de la violencia y la delincuencia.

\section{Tabla 4. Variable costo de la violencia y la delincuencia}

\begin{tabular}{lll}
\hline Variable & Dimensión & Indicadores \\
\hline Costo de la & Costo en el & $\bullet$ Costo por cantidad \\
violencia y la & sistema penal & de audiencias. \\
delincuencia & Costo en los & - Costo diario, \\
& privados de & mensual y anual por \\
libertad & cada interno en el \\
& & CCR. \\
& & Costo diario, \\
& mensual y anual de \\
& & 711 internos. \\
& & Comparación del \\
& & costo social de los \\
& & privados de libertad. \\
\hline
\end{tabular}

Fuente: elaboración propia de la investigación

Como muestra la tabla anterior, el estudio hace una estimación de los procesos penales y el costo de privación de libertad. El cálculo se realizó a través del método contable, analizando los costos directos: 1) al sistema de justicia, la estimación del costo del sistema de justicia penal, se realizó basada en los datos suministrados por la Fiscalía sobre la cantidad de 990 audiencias conocidas en el Tribunal de Atención Permanente; 627 en el Tribunal de la Instrucción y 299 el Tribunal Colegiado; en total 1916. Se asignó un costo de 30,000 pesos por audiencia, (Cruz, 2015) ${ }^{13}$.

2) El costo en el sistema penitenciario; costo de manutención de 711 internos en el CCR-15. Este costo se consideró basado en el costo diario de un interno, el cual se estima en 57 dólares $^{14}$; o sea, 2,736 pesos dominicanos los internos.

Para calcular el costo social de los privados de libertad, se estimó la producción económica no concebida por los internos del CCR-15, la que, según el Ministerio de Trabajo debe igualarse al sueldo mínimo inferior, equivalente a 7,800 pesos dominicanos. Además, se comparó el costo mensual de un interno con el sueldo mensual de un empleado con un salario mínimo. Se calculó el costo de un estudiante de primaria del sector público y en comparación con el costo de un interno.

En otro orden, se computó el costo total anual de manutención de los 711 internos, comparado con el costo de una carrera universitaria en la universidad Organización y Método, O \& M, la cual tiene un costo de 3,000 pesos mensual por matrícula de grado en diferentes carreras.

El costo social de los privados de libertad se realizó con la siguiente fórmula:

$C \boldsymbol{s}=s+j+(x)$

$\mathrm{Cs}=$ costo social de la delincuencia

$\mathrm{S}=$ Costo del sistema de justicia

$\mathrm{J}=$ Costo del sistema penitenciario

$\mathrm{X}=$ Pérdida social de beneficios de los recursos producidos por los privados de libertad.

\section{Resultados}

\section{Nivel de violencia y delincuencia}

A continuación, la tabla muestra los resultados de los diferentes tipos de delitos

14. Este dato fue ofrecido por personal interno del CCR-15.
13. Osvaldo de la Cruz, 2015. Hacer justicia implica un alto costo al Estado dominicano. Periódico El Dinero.

Recuperado de www.eldinero.com.do/14736/hacer-justicia-implica-un-alto-costo-al-estado-dominicano/ 
Tabla 5. Cantidad de delitos en 2017

\begin{tabular}{lcc}
\hline Delito & Cantidad & $\%$ \\
\hline Drogas & 311 & 31 \\
Robo & 269 & 27 \\
Violencia intrafamiliar & 176 & 18 \\
Homicidios & 60 & 6 \\
Golpes y heridas & 54 & 5 \\
Porte ilegal de armas & 47 & 5 \\
Abuso infantil & 5 & 0 \\
Agresión sexual & 31 & 3 \\
Seducción & 5 & 0 \\
Estafa & 6 & 0 \\
Abuso de confianza & 4 & 0 \\
Falsificación & 4 & 0 \\
Medio Ambiente & 4 & 0 \\
Otros delitos & 14 & 1 \\
Total & 990 & \\
\hline
\end{tabular}

Fuente: elaboración propia con base en el informe de la Fiscalía de La Romana, 2017

Como muestra la tabla anterior, al Sistema de Justicia Penal entraron 990 casos, de los cuales, 311 correspondían a la violación a la Ley de drogas, representando el $31 \%$. Por otro lado, el robo, en sus distintas modalidades; robo simple, atraco y asociación de malhechores tuvo un total de 269 casos, representando el 27\%. La violencia intrafamiliar tuvo 176 casos y representa el $18 \%$. En otro orden, hubo un total de 60 homicidios; los mismos representan el $6 \%$ de los casos penales. El tipo penal de golpes y heridas tuvo 54 con un 5\%; el porte ilegal de armas 47 casos, teniendo también 5\%. La agresión sexual, 31 casos y un 3\% de los casos. Los demás tipos penales obtuvieron entre 0 y $1 \%$ respectivamente.

A continuación se calcula la tasa de delitos cometidos contra la persona o propiedad.
Tabla 6 Tasa de delitos por cada 100 mil habitantes

\begin{tabular}{cc}
\hline Delitos & Tasa/\% \\
\hline Homicidios & 24 \\
Robos & 110 \\
Violencia intrafamiliar & 72 \\
Golpes y heridas & 22 \\
Delitos sexuales & 15 \\
Otros delitos & 13 \\
\hline
\end{tabular}

Fuente: Resultados de esta investigación, basada en informe de la Fiscalía de La Romana 2017

Como se puede verificar en la tabla anterior, el delito de robo en sus diferentes modalidades el tipo penal más elevado, con una tasa de $110 \%$ por cada 100 mil habitantes. Asimismo, la violencia intrafamiliar tuvo una tasa de $72 \%$ por cada 100,000 habitantes, en tanto que los delitos de golpes y heridas fueron de $22 \%$, y los delitos sexuales $15 \%$ por cada 100,000 habitantes. La tasa de homicidios fue de $24 \%$ por cada 100,000 habitantes

\section{Resultado 2. Condición de los privados de libertad}

Cantidad de presos preventivos y condenados

La tabla siguiente muestra la cantidad presos preventivos y la cantidad condenados

Tabla 7. Cantidad de presos preventivos y condenados

\begin{tabular}{cc}
\hline Privados de libertad & Total \\
\hline Preventivos & 695 \\
Condenados & 127 \\
\hline
\end{tabular}

Fuente: Elaboración propia, basado en cantidad de privados de libertad CCR-15 y cuartel del Palacio de Justicia 
Como muestra la tabla, el total de presos preventivos tanto en el CCR-15 y en el cuartel preventivo del Palacio de Justicia, fueron 695; y representan el $85 \%$ de los privados de libertad, mientas que los presos condenados fueron 127 , con el $15 \%$.

\section{Hacinamiento crítico}

La tabla a continuación muestra el nivel de hacinamiento del cuartel preventivo del Palacio de Justicia, desde 2013 a 2017.

Tabla 8. Hacinamiento crítico en el cuartel preventivo del Palacio de Justicia

\begin{tabular}{cccccc}
\hline & $\mathbf{2 0 1 3}$ & $\mathbf{2 0 1 4}$ & $\mathbf{2 0 1 5}$ & $\mathbf{2 0 1 6}$ & $\mathbf{2 0 1 7}$ \\
\hline Cantidad & 98 & 95 & 96 & 123 & 112 \\
Hacinamiento & 490 & 475 & 480 & 351 & 320 \\
\hline
\end{tabular}

Fuente: Resultados de esta investigación

Como muestra la tabla anterior, el cuartel preventivo del Palacio de Justicia ha presentado una tasa muy alta de hacinamiento durante varios años. En ese sentido, la capacidad de alojamiento durante los años 2013 a 2015 era de aproximadamente para 20 personas; sin embargo, la cantidad de privados de libertad en condiciones preventivas era casi de 100 , lo que determina que la sobrepoblación en ese periodo fue casi 500\%. Durante los ańos 2016 y 2017 la sobrepoblación tuvo un nivel de 351\% y $320 \%$.

\section{Resultado 3. Costo de la delincuencia en la provincia de La Romana.}

La tabla a continuación muestra el costo diario, mensual y anual de cada interno y el costo total de los 711 internos en el CCR-15 en 2017.
Tabla 9 Costo por interno en el CCR La Romana

\begin{tabular}{ccccc}
\hline Costo & $\begin{array}{c}\text { Indivi- } \\
\text { dual en } \\
\text { pesos }\end{array}$ & $\begin{array}{c}\text { Indivi- } \\
\text { dual en } \\
\text { dólares }\end{array}$ & $\begin{array}{c}\text { Total en pesos } \\
\text { por 711 priva- } \\
\text { dos de libertad }\end{array}$ & $\begin{array}{c}\text { Total en } \\
\text { dólares } \\
\text { por 711 } \\
\text { privados de } \\
\text { libertad }\end{array}$ \\
\hline $\begin{array}{c}\text { Diario } \\
\text { Men- }\end{array}$ & 2,736 & 57 & $1,945,296$ & 34.128 \\
sual & 82,080 & 1.710 & $58,358,88$ & 1.215 .810 \\
Anual & 984960 & 20520 & $700,306,560$ & 14.589 .720 \\
\hline
\end{tabular}

Fuente: Estimación propia basada en dato de esta investigación

Como muestra la tabla, el costo anual de un imputado fue RD\$984,960, o sea, 20.520 dólares. Esta cifra refleja que el costo mensual de un interno supera diez veces el sueldo mínimo inferior de un empleado.

El costo de manutención de un interno equivale a la inversión de 20 estudiantes de primaria o secundaria de sector público. Se estima que con el costo de 711 privados de libertad, se pudo haber pagado la matrícula de 19,453 estudiantes de grado por un ańo de en la universidad Dominicana $\mathrm{O}$ y $\mathrm{M}$.

La tabla siguiente muestra el costo en el Sistema de Justicia Penal, manutención en el Sistema Penitenciario y el costo social de los privados de libertad.

Tabla 10 Costo de la delincuencia en la provincia de La Romana, 2017

\begin{tabular}{ccc}
\hline & Total en pesos & Total en dólares \\
\hline Sistema de Justicia & $57,480.000$ & 1.1975 .00 \\
$\begin{array}{c}\text { Manutención Sistema } \\
\text { Penitenciario }\end{array}$ & $700,306,560$ & 14.589 .720 \\
$\begin{array}{c}\text { Dinero no percibido por } \\
\text { privados de libertad } \\
\text { Total }\end{array}$ & $5,545,800$ & 115.531 \\
\hline
\end{tabular}

Fuente: Estimación propia basada en dato de esta investigación 
En la tabla muestra que hubo un costo estimado de $\mathrm{RD} \$ 57,480,000$ en los servicios judiciales de los casos penales de la provincia de La Romana; el dinero no percibido por privados de libertad fue $\mathrm{RD} \$ 5,545,800$. La suma refleja un costo de RD\$ $763,332,360$.

\section{Conclusiones y recomendaciones}

\section{Violencia y delincuencia}

De acuerdo con los resultados de este estudio, se ha podido comprobar que la provincia de La Romana tiene un alto índice de violencia y delincuencia. Se encontró una tasa de homicidios de $24 \%$ por cada 100,000 habitantes. Según la literatura, el homicidio constituye uno de los indicadores más completos, comparables y precisos para medir la violencia. (Oficina de las Naciones Unidas contra la Droga y el Delito, 2014). De acuerdo a la literatura analizada, este dato comprueba que la provincia de La Romana tiene un carácter epidémico de violencia (Centro de las Naciones Unidas Regional para la Paz, el Desarme y el Desarrollo en América Latina y el Caribe, 2017).

Cabe resaltar que la fuente consultada no detalla la cantidad por tipos de homicidios; cuántos se relacionaron a otras actividades delictivas y cuantos estaban asociados a conflictos interpersonales. (Oficina de las Naciones Unidas contra la Droga y el Delito, 2013). Sin embargo, aunque no se muestre cuáles fueron las causas de los homicidios, se encontró un alto índice de violencia interpersonal. En relación a esto, la violencia intrafamiliar tuvo una tasa de $72 \%$; los delitos de golpes y heridas obtuvieron una tasa de $22 \%$ y los delitos sexuales tuvieron una tasa $15 \%$ por cada 100,000 habitantes respectivamente. La suma de estos delitos muestra que la violencia interpersonal obtuvo una tasa de $117 \%$ por cada 100,000 habitantes.

Por otro lado, se encontró que el $31 \%$ de procesos ingresados al sistema correspondían a la violación de la Ley de droga en sus diferentes calificaciones. Además, se demostró que el delito de robo tuvo una tasa de $110 \%$ por cada 100,000 habitantes.

Es importante aclarar que este estudio solo se basa en los casos ingresados al sistema de justicia penal y excluye las denuncias realizadas en la policía y las encuestas de victimización y percepción de la violencia y la delincuencia.

\section{Resultados sobre la condición de los presos preventivos y el respeto de sus Derechos}

Se encontró que solo el $15 \%$ de los hombres privados de libertad tenían una condena, mientras que el $85 \%$ eran preventivos. Este dato supera lo encontrado en la literatura, la cual afirma que "los expedientes de gran parte de la población en reclusión, casi el $50 \%$ son procesados" (Comisión Nacional de los Derechos Humanos, 2015). En ese mismo orden, estudio nacional sobre la condición de prisión reveló que en 2014, el 59\% de los privados de libertad cumplían prisión preventiva, mientras que el $41 \%$ habían sido condenados (Oficina Nacional de Defensa Pública, 2014). Los resultados confirman que el hacinamiento en la provincia de La Romana se debe un excesivo uso de la prisión preventiva. (Barros Leal, 2014). Además se evidencia que la sobrepoblación carcelaria se debe a que el sistema de justicia penal de La Romana es deficiente, por lo cual hay demora en los procesos (Noel Rodríguez, 2015).

Por otro lado, este estudio encontró que el destacamento preventivo del Palacio de Justicia ha mantenido una sobrepoblación crítica durante varios años. Según lo descrito en la literatura, la sobrepoblación carcelaria se refiere a que la densidad penitenciaria es mayor que 100; cuando la densidad es igual o mayor que 120 , se considera sobrepoblación crítica o hacinamiento crítico. (Carranza, 2012). Con base a lo anterior, la tasa de sobrepoblación en el destacamento del Palacio de Justicia desde 2013 a 2017 fue de 320\%, 
mínima y 490\%. Este hallazgo coincide con estudios realizados por la Oficina de la Defensa Pública, la cual confirma que en 2014 hubo una sobrepoblación carcelaria entre 112\% y 570\% en las cárceles públicas del país. Otros estudios revelan las cárceles de América Latina y El Caribe están sobrepobladas y hacinadas (Comisión Interamericana de Derechos Humanos, 2011).

\section{Costo de la violencia y la delincuencia}

Se pudo determinar que el costo del sistema de justicia penal por 990 audiencias realizadas en 2017, más el costo de manutención de711 internos y el costo social no percibido por los privados de libertad, se estima en $\operatorname{RD} \$ 763,332,360$, equivalente a USD 17.173.670. Se calculó que el costo anual de un imputado o interno, es de $\mathrm{RD} \$$ 984,960, o sea, 20.520 dólares. Esta cifra refleja que el costo anual de un imputado equivale a la inversión hecha a 20 estudiantes de primaria o secundaria del sector público.

Otra estimación refleja que, con el costo de manutención de los 711 privados de libertad, se pudo pagar la matrícula de 19,453 estudiantes en la universidad Dominicana $\mathrm{O}$ y $\mathrm{M}$ durante un año. Esta estimación corrobora con estudios realizados, que muestran en 2002 el costo de una carrera universitaria por personas en 15 Estados de Estados unidos era de 9.454 dólares en un año, mientras que el costo de un año de reclusión por persona era de 24.809 dólares (Dammert \& Díaz, 2005).

En otro orden, se puede evidenciar que el costo mensual de un interno supera diez veces el sueldo mínimo de un empleado. Este dato es confirmado por otro autor, el cual afirma que en Estados Unidos, el sueldo mínimo era 28.3 dólares diarios, mientras que el costo diario por recluso era de 65 dólares en 2003; mientras que en Inglaterra, el sueldo mínimo era 67,4 dólares diarios y el costo de encarcelamiento por recluso era de 166 dólares; asimismo, en Australia el sueldo mínimo diario era de 63,4 y era de 84 dólares diarios en 2003 (Dammert \& Díaz, 2005).

Este estudio excluye el costo directo de victimización la pérdida material y las lesiones de la víctima, la consecuencia de la discapacidad, días de trabajo perdido y la vida productiva de quienes han muerto. Tampoco se calculan los costos de atención médica, ni costos de seguridad privada, pérdida de oportunidades de trabajo ni pérdidas materiales. (Kliksberg, 2007)

En resumen, se pudo comprobar que:

1. La Romana tiene un alto índice de violencia y delincuencia

2. El sistema penal de esta provincia es deficiente mostrado en el alto porcentaje de presos preventivos.

3. Hay un alto nivel de sobrepoblación en el destacamento preventivo del Palacio de Justicia, lo cual trae consecuencias violación de los Derechos Humanos de los privados de libertad.

4. Se mostró que hay gasto muy alto en el sistema de justicia y penitenciario.

Con base a lo anterior, es necesario que la provincia de La Romana implemente proyecto de prevención de la violencia y la delincuencia. Se ha determinado que para disminuir la violencia y la delincuencia es necesario desarrollar políticas de prevención y que éstas se apliquen en los tres niveles de intervención; primario, secundario y terciario.

Por otro lado, es necesario optimizar el sistema de justicia penal a los fines de reducir el porcentaje de presos preventivos, prevenir la sobrepoblación carcelaria y disminuir el costo en el sistema penal y penitenciario.

\section{Referencias}

Arias, F. (2012). El proyecto de Investigacion. Caracas: Editorial Episteme. 
Arriagada, I., \& Godoy, L. (2000). Prevenir o reprimir: falso dilema de la seguridad ciudadana. Santiago de Chile: Cepal.

Banco Interamericano de Desarrollo. (2015). Los costos del crimen y la violencia en el bienestar en America Latina y El Caribe. New York: BID.

Banco Interamericano de Desarrollo. (2017). Los costos del crimen y la violencia. Nuevos hayazgos en America Latina y El Caribe. Nueva York: BID.

Barros Leal, C. (2014). La pena de prisioón preventiva en América Latina: Los privados de libertad y sus derechos humanos. Revista Instituto Brasileño de Derechos Humanos. Obtenido de http://milas.x10host.com/ojs/index.php/ibdh/ article/view/265

Carranza, E. (2012). Situación penitenciaria en América Latina y el Caribe ¿Qué hacer? Anuario de Derechos Humanos 2012. Obtenido de https:// anuariocdh.uchile.cl/index.php/ADH/article/ download/20551/21723/0

Centro de las Naciones Unidas Regional para la Paz, el Desarme y el Desarrollo en América Latina y el Caribe. (2017). Estudio Normativo: La necesaria vinculación entre las normas sobre violencia por motivos de género y las normas de regulación y control de armas pequeñas. Perú: UNLIREC. Obtenido de http://www.unlirec.org

Comisión Interamericana de Derechos Humanos. (2011). Informe sobre los derechos humanos de las personas privadas de libertad en las Américas. OEA. Obtenido de https://www.oas.org/es/cidh/ppl/ docs/pdf/ppl2011esp.pdf

Comisión Nacional de los Derechos Humanos. (2015). La sobrepoblación en los Centros Penitenciarios de la Repúblca Méxicana. $C N D H$ Mexico.

Corporación Andina de Fomento. (2014). Por una América Latina más segura: Una nueva perspectiva para prevenir y controlar el delito. Bogotá, Colombia: CAF.

Cruz, O. d. (27 de julio de 2015). Hacer justicia implica un alto costo al Estado dominicano. El Dinero. Obtenido de https://www.eldinero.com. do/14736/hacer-justicia-implica-un-alto-costo-alestado-dominicano/

Dammert, L., \& Díaz, J. (2005). El Costo de Encarcelar. Programa de Seguridad y CiudadaniaFLACSO-Chile. Obtenido de http://www. flacsochile.org/wp-content/uploads/2015/05/Elcosto-de-Encarcelar.pdf

Hernandez Sampieri, C. F. (2010). Medodologia de la Investigacion. 5ta. edicion. Mexico: McGRAWN HILL.

Instituto Interamericano de Derechos Humanos. (2011). Derechos humanos, seguridad ciudadana y funciones policiales. San José, C.R: EditorialServicios Especiales del IIDH.

Kliksberg, B. (2007). Mitos y realidades sobre la criminalidad en America Latina. Algunas anotaciones estrategicas sobre como enfrentar y mejorar su cohesion social. Union Europea: FIIAPP.

Latinobarómetro. (2016). Informe 2016. Buenos Aires: BAnco Intereramericano de desarrollo.

Ministerio de Economía, Planificación y Desarrollo. (2016). Plan para el desarrollo económico local de la provincia La Romana. La Romana: Ministerio de Economía, Planificación y Desarrollo.

Ministerio de Economía, Planificación y Desarrollo, Unidad Asesora de Análisis Económico y Social. (2014). El Mapa de la pobreza en la República Dominicana 2014. Informe general. Santo Domingo: Ministerio de Economía, Planificación y Desarrollo.

Ministerio de Educación de la República Dominicana. (2018). Informe sobre la situación y evolución de la ejecución presupuestaria 2017. Santo Domingo: MINERD. 
Muñoz Razo, C. (2011). Cómo elaborar y asesorar una investigación de tesis. México: PEARSON EDUCACIÓN.

Naciones Unidas. (2014). Manual Sobre Estrategias Para Reducir el Hacinamiento en las Prisiones. Neva York: UNODC.

Noel Rodríguez, M. (2015). Hacinamiento Penitenciario en América Latina: Causas y Estrategias para su Reducción. México: CNDH.

Observatorio Regional de Justicia Penal Juvenil. (2015). Informe de Monitoreo a los Sistemas de Justicia Penal Juvenil en América Latina. Bolivia: Gráfica "J.V." Editora.

Oficina de las Naciones Unidas contra la Droga y el Delito. (2010). Recopilación de reglas y normas de las Naciones Unidas en la esfera de la prevención del delito y la justicia penal. New York: Naciones Unidas.

Oficina de las Naciones Unidas contra la Droga y el Delito. (2013). Estudio Mundial sobre El Homcidio. Resumen Ejecutivo. Obtenido de https:// www.unodc.org/documents/gsh/pdfs/GLOBAL_ HOMICIDE_Report_ExSum_spanish.pdf

Oficina de las Naciones Unidas contra la Droga y el Delito. (2014). Estudio Mundial sobre el Homicidio. UNODC. Obtenido de https://www.unodc.org/ documents/gsh/pdfs/GLOBAL_HOMICIDE_ Report_ExSum_spanish.pdf

Oficina Nacional de Defensa Pública. (2014). Informe Anual. Santo Domingo: Editora Corripio, S.A.S.
Oficina Nacional de Estadísitica. (2016). Perfiles Estadísticos Provinciales. Provincia La Romana. Oficina Nacional de Estadística.

Organización Mundial de la Salud. (2014). Informe Sobre la Situacion Mundial de la Prevencion de la Violencia. Washington, D.C.: OMS. Obtenido de apps.who.int/iris/bitstream/10665/145089/1/ WHO_NMH_NVI_14.2_spa.pdf

Organización Mundial de la Salud. (2014). paises de América Latina y el Caribe tienen las tasas de homicidio más altas del mundo. Obtenido de www.unmultimedia.org/.../ oms-paises-de-america-latina-y-el-caribe-tiene

Organización Mundial de la Salud. (14 de Septiembre de 2016). Informe sobre la situación mundial de la prevención de la violencia 2014. Washington, DC: Organización Mundial de la Salud. Obtenido de http://www.who.int/ mediacentre/factsheets/fs239/es/

Procuraduría General de la República. (2018). Informe 2017. Santo Domingo.

Programa de las Naciones Unidas para el Desarrollo. (2014). Informe Regional de Desarrollo Humano 2013-2014. Seguridad Ciudadana con rostro humano. Diagnostico y propuesta para America Latina. Panama: Alfa Omega Impresiones.

Seguridad, Justicia y Paz. (2018). Metodología del ranking (2017) de las 50 ciudades más violentas del mundo. México. Obtenido de https://www. seguridadjusticiaypaz.org.mx/biblioteca/prensa/ category/6-prensa 


\section{Datos de filiación}

Magnolia Ortiz Rodríguez. Candidata a doctora en Proyectos, Universidad Internacional Iberoamericana UNINI México. Línea de investigación: Proyectos Integrados. Tiene una maestría Psicología Clínica, por la Universidad UTESA. Cursos y especialidades: Evaluación de Impacto de Proyectos Sociales, J-PAL; Técnicas Cuantitativas y Cualitativas de la Investigación, Universidad Politécnica de Valencia; gestión de Proyectos de Desarrollo, Banco Interamericano de Desarrollo; aspirante a Trabajadora Social, Escuela Nacional de la Judicatura; intervención Social en protección al menor, Universidad Ryerson, Canadá, y la Facultad de Ciencias Económicas y Sociales de la Universidad Autónoma de Santo Domingo. Corre-e: magnolia_ortiz06@hotmail.com 